\title{
MORPHOLOGIE VÉGÉTALE ET RÉPARTITION DES MAMMIFÈRES
}

\author{
par \\ MARIE-CHARLOTTE SAINT GIRONS

\begin{abstract}
Muséum national d'Histoire naturelle, Laboratoire d'Ecologie générale, 4 Avenue du Petit Château, 91800 Brunoy, France
\end{abstract}

\begin{abstract}
RESUME
Dans beaucoup de travaux concernant la répartition des Mammifères, la végétation est étudiée tout particulièrement en tant que source de nourriture et l'importance de la morphologie des plantes est laissée de côté. La morphologie végétale n'est un facteur limite que dans le choix de la „,niche écologique" mais n'intervient généralement pas dans la répartition géographique. En revanche, la densité des Mammifères est liée dans de nombreux cas à la morphologie de la végétation, particulièrement pour les mangeurs d'herbe (Rongeurs), moins pour les Insectivores et les petits Carnivores. De nombreux exemples sont donnés concernant les Mammifères d'Europe et d'Afrique du Nord; ils concernent la liaison entre la morphologie des plantes et les nids, le couvert, les points de repères assurés par les plantes, les exceptions et les adaptations, particulièrement dans les îles.
\end{abstract}

\section{SUMMARY}

In many studies on mammalian distribution, vegetation is considered in particular as a source of food and its importance in terms of habitat has been neglected. The type of vegetation cover is a limiting factor only in the choice of ecological niches but does not in general affect the overall distribution. On the contrary, the density of mammals is in many cases associated with the morphology of the plant cover, especially in the case of granivores (rodents), less for insectivores and small carnivores. In the paper several examples are given concerning mammals of Europe and North Africa and the importance of vegetation morphology for nests, cover and landmarks, as well as exceptions and adaptations, especially on islands.

\section{INTRODUCTION}

La distribution de nombreux Mammifères est liée à la couverture végétale. On connaît des espèces steppiques, d'autres sont forestières mais, dans la plupart des cas, une analyse détaillée des rapports entre un Mammifère et son milieu végétal n'a pas été entreprise, à l'exception des rapports trophi- ques. On a assez souvent noté toutefois la coïncidence de l'aire de répartition d'un Mammifère avec celle d'une espèce végétale. Dans ce cas, les mammalogistes se bornent à juste titre à constater la similitude et n'établissent pas de relations hypothétiques (Fons, 1976 pour Suncus etruscus et l'association du Chêne vert). La recherche du facteur limite qui conditionne soit la répartition dans un sens large soit le choix de la niche écologique reste le plus souvent à faire. En outre, tel élément du milieu végétal, facteur limite sous certaines conditions climatiques ou édaphiques, verra s'amenuiser son importance si la latitude, le sol ou encore les façons culturales changent. Tous les naturalistes de terrain se sont heurtés à ces problèmes. Lors de nos recherches dans la nature, nous avons à maintes reprises remarqué l'importance que joue la morphologie végétale dans la répartition locale des diverses espèces. C'est une notion qui pourtant reste le plus souvent vague dans les travaux d'écologie de Mammifères, peut-être parce qu'il est difficile de cerner les liens complexes unissant les animaux, et tout particulièrement les petites espèces, au couvert végétal. Dans les pages suivantes, nous tentons de définir ces liens à l'aide d'exemples le plus souvent personnels, s'appliquant à des espèces que, sauf exceptions (Castor, Chat sauvage), nous avons observées maintes fois dans la nature. Ce sont essentiellement des Mammifères d'Europe occidentale et d'Afrique du Nord.

Cette étude résulte de multiples séries de piégeages, d'observations dans la nature et aussi de l'uti- 
lisation du matériel fourni par l'analyse des pelotes de Rapaces, particulièrement précieux pour connaître les limites géographiques d'une aire de répartition. Les grands Mammifères ne figurent pas dans ces pages. Leur répartition comme le choix de leur niche écologique dépend souvent plus de la pression humaine que du besoin spécifique d'une morphologie végétale déterminée. Nous entendons le terme "morphologie végétale" dans son sens large de strates de végétation (herbacée, arbustive, arborée), en y joignant les qualificatifs de densité (touffue, clairsemée, fermée, ouverte etc...) et sans préjuger des espèces qui les constituent.

\section{VEGETATION SUPPORT ET SOUTIEN DE L'ABRI}

1.1. Abri toujours dans les arbres ou les arbustes Quelques Mammifères utilisent un nid exclusivement aérien, disposé dans la strate arborée. Celui de l'Ecureuil roux, Sciurus vulgaris, est bien connu. La présence d'un arbre est indispensable à l'espèce. Au contraire, celle des strates arbustive et herbacée n'est pas nécessaire. Il y a des Ecureuils dans les futaies sans sous-bois et ils habitent aussi bien les bois clairsemés que les bouquets d'arbres des parcs ou les forêts denses. Leur densité plus accentuée en forêt de Conifères est en liaison avec la nourriture (graines des cônes en hiver) plus qu'avec la morphologie du support.

\subsection{Abri de préférence dans les arbres ou les ar- bustes}

\subsubsection{Les arbres}

Certaines espèces réputées arboricoles ne sont pas en fait strictement liées à la présence d'une strate arborée. C'est le cas de deux Gliridae de la faune européenne: le Loir, Glis glis, et le Lérot, Eliomys quercinus. L'un et l'autre se construisent des nids dans les arbres, le Loir souvent un peu plus haut que le Lérot ( 4 à $6 \mathrm{~m}$ au-dessus du sol pour le Loir d'après Kahmann, 1951). On les rencontre également dans les trous de Pics et ils peuvent fréquenter les constructions en toutes saisons. Le Loir hiberne non dans un abri aérien mais dans un nid souterrain à un peu moins d'un mètre de profondeur, là où il est assuré de bénéficier de condi- tions thermiques sensiblement constantes. Il le creuse lui-même (Koenig, 1960) ou utilise une grotte naturelle. On l'a trouvé à plus de $100 \mathrm{~m}$ d'orifices de grottes dans les terrains calcaires du Vercors. Quant au Lérot, il s'endort dans des cavités naturelles, des anfractuosités de murs, parfois des arbres creux en région dépourvue de rochers et de constructions, voire des nichoirs à Oiseaux. La présence de l'arbre n'est pas indispensable et la strate arborée ne constitue donc pas un facteur limitatif de la répartition locale.

\subsubsection{Les arbustes}

Le cas du Muscardin, Muscardinus avellanarius, est différent. Son nid d'été est accroché dans les buissons, souvent à moins d'un mètre de hauteur (Kahmann, 1951). Nous l'avons observé à l'aisselle des branches de jeunes Conifères en Carinthie. Cette espèce ne creuse pas et ne fréquente ni les cavités ni les constructions, à notre connaissance du moins. La présence d'une strate arbustive dense, surmontée ou non d'une strate arborée, lui est indispensable en été. Nous n'avons pas vu son nid d'hiver mais sa présence plusieurs fois signalée dans les nichoirs à Oiseaux et les arbres creux conduit à penser qu'il abandonne à l'automne la strate arbustive pour s'endormir dans les cavités des arbres à une hauteur que nous ne saurions préciser. C'est donc un exemple d'une espèce liée selon toute vraisemblance à l'existence de deux strates de végétation. Ceci permettrait d'expliquer sa rareté en dehors des grandes forêts, par exemple dans les zones découvertes de l'ouest où pourtant sa présence est signalée sporadiquement. S'il existe en forêt de Rennes (Le Garff, in verbis), nous l'avons vainement recherché dans le bocage, une centaine de kilomètres au sud (fig. 1). La présence simultanée des deux strates, arborée et arbustive, serait donc un facteur indispensable de sa répartition.

La forme sauvage du Rat noir, Rattus rattus frugivorus, fréquente également la strate arbustive en région méditerranéenne. Ce Rat partage la niche écologique du Lérot dans le sous-bois touffu des forêts du Maroc septentrional (observation entre Rabat et Casablanca). Toutefois, sa plasticité comportementale est telle qu'on peut le rencontrer dans bien d'autres milieux et la liaison avec l'arbuste est loin d'être rigide. 


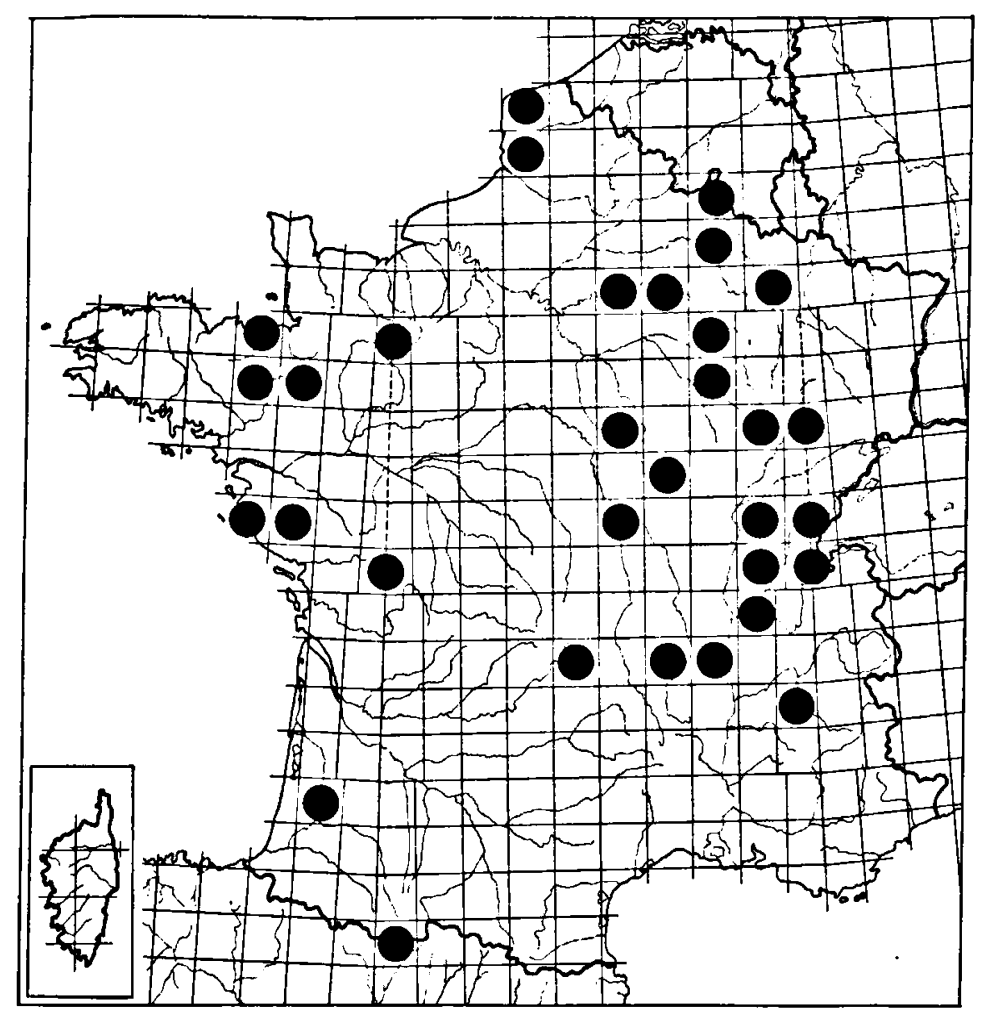

Fig. 1. Répartition de Muscardinus avellanarius en France.

\subsection{Abris souterrains}

1.3.1. Présence indispensable des racines comme soutien

Ce cas est particulièrement réalisé dans les régions semi désertiques sableuses. On sait que la survie des espèces désertiques est liée à l'utilisation de l'eau. Pour assurer en toutes saisons une économie maximale des ressources en eau, les Mammifères sont nocturnes et passent la journée dans les abris souterrains aux conditions microclimatiques quasi constantes (température modérée, humidité forte). Or ces abris souterrains ne peuvent être creusés qu'en terrain meuble mais pourtant suffisamment compact pour éviter les éboulements. Les buissons des zones sableuses (ergs morts, dayas) assurent cette compacité en retenant le sable autour de leurs racines et de leurs basses branches plus ou moins enfouies. Petter (1953) a bien établi la liaison entre les terriers d'un Rongeur, Meriones libycus, et l'existence de buissons d'une Rhamnacée, le Jujubier, Zizyphus lotus. En outre, ces buissons en formation toujours très ouverte, constituent une source de nourriture et un abri contre les prédateurs. Les buttes à Jujubier du sud marocain sont truffées d'orifices de terriers et c'est toujours là que les rendements de piégeage sont les meilleurs. C'est donc un exemple de facteur limite de la répartition locale constitué par la strate arbustive.

\subsubsection{Racines utilisées comme point d'appui mais} non indispensables

Les petits Mammifères des zones tempérées disposent le plus souvent de sols suffisamment compacts pour creuser leurs galeries. Toutefois, la plupart d'entre eux utilisent les racines comme point d'appui chaque fois que cela leur est possible. Ce tygmotactisme est différemment accentué suivant les espèces. Le Campagnol des champs, Microtus arvalis, en est, par exemple, à peu près totalement dépourvu, sauf peut-être en captivité où ses galeries s'appuient aux parois rigides des enclos. Des espèces sylvicoles comme le Campagnol roussâtre (Cletbrionomys glareolus) et surtout les Mulots (Apodemus sylvaticus et Apodemus flavicollis) utilisent lorsque cela leur est possible l'appui des 
racines. Jennings (1975) a montré la liaison du réseau de galeries d'Apodemus sylvaticus avec les racines de Noisetiers, Coryllus avellana. Il est probable que l'arbrisseau joue le rôle d'étai mais celui de protection des orifices, voire de source de nourriture, vient s'y ajouter. Dans les haies plantées à plat, nous n'avons jamais trouvé d'entrée de terrier permanent de Mulot au delà de la limite du rideau de végétation. De même le Blaireau, Meles meles, creuse ses terriers sur des pentes souvent accentuées, là où les racines permettent d'épauler les galeries. On ne le trouve pas sur les pentes gazonnées.

\section{VEGETATION EN TANT QUE MATERIAU POUR L'ABRI}

\subsection{Abris naturels}

Il existe des abris naturels dans les troncs d'arbres creux. Ceux évidés par le Pic noir, Dryocopus martius, sont particulièrement grands et à orifice ovale bien visible. On pourrait écrire qu'il s'agit là d'abris préfabriqués pour les Mammifères. Ils constituent pour certains des gîtes d'hiver occasionnels ou systématiques mais d'autres leur sont encore plus étroitement inféodés; les Chauves-Souris arboricoles utilisent ces cavités en toutes saisons et ne peuvent fréquenter régulièrement un milieu dépourvu d'arbres. C'est un nouveau cas de liaison étroite entre la répartition d'une espèce et la présence de la strate arborée qui agit comme facteur limite aussi bien de la répartition locale que géographique.

\subsection{Abris tissés}

Des Rongeurs de petite taille utilisent la végétation herbacée pour se confectionner des nids suspendus à faible hauteur au-dessus du sol. Ces abris tissés sont particulièrement le fait du Rat des moissons, Micromys minutus. Ce très petit Muridae passe l'hiver au niveau du sol, sous la litière, parfois dans un tunnel superficiel et peu profond, souvent aussi dans les meules. Pour confectionner ses nids d'été, le Rat des moissons utilise les feuilles de monocotylédones dilacérées en bandes de 1 à $2 \mathrm{~mm}$ de largeur. Ces rubans, encore attachés à la plante, forment une sorte de trame que le Rongeur consolide avec les matériaux dont il peut disposer. L'ensemble est suspendu le plus souvent à mi hauteur de la tige qui le soutient. L'existence d'une végétation herbacée de taille élevée lui est donc nécessaire au moment de la saison de reproduction. Pour le reste, il s'accommode de n'importe quel habitat. De ce fait, sa niche écologique est constituée par les marais et roselières non inondés en permanence et, secondairement, par les champs de céréales. En France, par exemple, il pourrait habiter un peu partout, même en l'absence de zones humides. Nous l'avons trouvé dans les hautes herbes bordant les chemins, les lisières de bois, les prairies de fauche et bien entendu les champs de céréales. Pourtant, sa densité est variable d'une région à l'autre et la proportion qu'il atteint dans le régime d'un Rapace nocturne comme l'Effraie varie de 0 à environ 30 pour mille proies dénombrées. Si on trace une carte de ces variations de densité, on s'aperçoit qu'elles correspondent aux variations d'importance des surfaces cultivées en maïs (maïs en grains dans le Sud-Ouest, maïs fourrage à l'ouest). En éliminant les départements riches en zones humides (Ain, Bouches-du-Rhône), on constate que la proportion la plus forte est notée pour ceux où la superficie des terres cultivées en maïs dépasse $20 \%$ de la surface totale. Au contraire, ceux où la proportion du Rat des moissons dans les proies s'avère faible ou nulle ont moins de $10 \%$ et généralement même moins de $1 \%$ de leur surface occupée par le mais (tab. I). Ces

\section{TABLEAU I}

Liaison entre l'importance des surfaces cultivées en maïs dans quelques départements et la proportion du Rat des moissons $(\% / 00)$ dans les pelotes de Tyto alba.

\begin{tabular}{lcc}
\hline $\begin{array}{l}\text { Plus de 20\% } \\
\text { en maïs }\end{array}$ & $\begin{array}{c}\text { Nombre de proies } \\
\text { déterminées }\end{array}$ & $\begin{array}{c}\text { \% oo du Rat des } \\
\text { moissons }\end{array}$ \\
\hline $\begin{array}{l}\text { Landes } \\
\text { Pyrénées-Atlantiques }\end{array}$ & 4807 & 31,2 \\
Eure-et-Loir & 8098 & 17,4 \\
& 795 & 17,6 \\
Moins de 1\% en maïs & & \\
Cantal & 858 & 1,0 \\
Corrèze & 826 & 6,0 \\
Creuse & 1942 & 6,2 \\
Gard & 2709 & 0,4 \\
Haute-Loire & 234 & 0 \\
Hérault & 253 & 0 \\
Lozère & 1064 & 0 \\
Var & 290 & 0 \\
Vaucluse & 2344 & 5,1 \\
\hline
\end{tabular}


proportions sont faussées dans les départements de l'ouest où manque la proie essentielle de l'Effraie, Microtus arvalis. C'est ainsi que le chiffre de 39 pour mille est atteint dans le Morbihan qui a moins de $10 \%$ de sa surface cultivée en maïs fourrage mais où le Rat des moissons fait partie des proies de remplacement de l'Effraie. Le Campagnol des champs fait également défaut dans la forêt landaise. La France, cinquième producteur mondial en 1973 consacre environ 2 millions d'hectares de terre au maïs. Cette culture présente sans doute pour le Rat des moissons des avantages supérieurs aux autres céréales du fait que les plants restent sur le sol plus longtemps que le blé et qu'en outre la végétation plus dense et plus haute assure un meilleur abri, recréant presque la morphologie des roselières. L'espèce s'était très raréfiée dans le bocage et avait pratiquement disparu du régime des Effraies il y a quelques décennies par introduction, sans doute, des moissonneuses-batteuses et des botteleuses en même temps que disparaissaient les meules et moyettes. Le Rat des moissons a reparu en nombre au moment du développement récent de la culture du maïs. Il est très probable qu'il y a là plus qu'une coïncidence et qu'il existe une liaison entre les deux phénomènes. A notre avis, la présence de cultures de maïs, surtout de maïs-grain coupé plus tard en automne, au moment où les jeunes de l'année se sont dispersés, ne serait un facteur limite de répartition locale que dans les régions dépourvues de biotopes morphologiquement comparables (est du Massif-Central) et constituerait partout un facteur favorable au développement de fortes populations (facteur de densité). Ce n'est vraisemblablement pas un facteur limite de la répartition géographique.

\subsection{Abris tapissés}

Pour être probablement le plus caractéristique, le cas du Rat des moissons n'est pas l'unique exemple d'utilisation par les petits Mammifères de la couverture végétale comme matériau pour confectionner l'abri. On peut citer aussi bien l'Ecureuil que le Muscardin pour ses nids d'été. Presque tous les Rongeurs entraînent dans leurs abris des brins d'herbe pour en tapisser leur nid. Ce comportement débute très rapidement lorsqu'un abri est adopté. Nous avons vu des Mulots relâchés loin de leur habituel terrain de chasse, chercher refuge dans un trou, orifice probable d'ancien terrier, et commencer immédiatement à l'aménager en coupant les brins d'herbe près de l'entrée et en les emportant à l'intérieur. Les espèces hibernantes sont, de ce point de vue, les plus liées à la morphologie végétale, toute interférence avec la thésaurisation de nourriture étant exclue. On connaît la tonte des prairies d'altitude par les Marmottes, Marmota marmota, pour tapisser l'abri d'hivernage de la tribu. Le Hérisson lui-même se ménage un gîte d'hiver dans des feuilles mortes accumulées. Le rôle de la strate herbacée apparaît donc le plus important dans le cas des abris tapissés mais il n'est sans doute jamais essentiel. Il serait hasardeux de parler ici de facteur limite de la répartition.

\subsection{Abris construits}

Il existe enfin des abris construits de toutes pièces par les animaux. Ils sont l'œuvre dans nos pays des Castors et des Rats musqués. En ce qui concerne Castor fiber, Richard (1964) a noté sa liaison étroite avec la présence de l'arbre dont le bois constitue le matériau indispensable à la construction des barrages et des huttes. Le Castor utilise surtout les Saules et les Peupliers, peut-être parce qu'ils constituent l'essentiel des espèces bordant les cours d'eau et que l'animal ne va pas très loin chercher un arbre à abattre. Le comportement constructeur du Rat musqué, Ondatra zibethicus, est, semble-t-il, plus complexe. Il peut bâtir de véritables huttes avec la végétation avoisinant la partie de l'étang où il s'est établi et n'y mêle jamais de boue (Meeuwissen, 1948 en Campine belge). On connaît également des huttes formées au départ d'une simple accumulation de reliefs de repas sur les radeaux-réfectoires. Celle-ci se transforme peu à peu par apport volontaire de matériel (et non de restes de nourriture) en un amas végétal en forme de dôme, creusé ou non d'une galerie alors que son constructeur continue à habiter les terriers aménagés dans la rive (Darchen \& Lecomte, 1959; Richard, 1962). Le Castor et le Rat musqué sont donc deux espèces liées au couvert végétal de leur niche écologique pour une partie au moins de leur comportement normal: présence d'arbres de dimensions données pour le Castor, d'herbes aquatiques pouvant être faucardées pour le Rat musqué. Pour- 
tant, dans un cas comme dans l'autre, les rôles respectifs de recherche de nourriture et de quête de matériau de construction se trouvent imbriqués et difficiles à délimiter. De plus, les schémas comportementaux sont adaptables et des Castors peuvent se réfugier dans des terriers et ne construire ni hutte ni barrage alors que des Rats musqués sont susceptibles de délaisser la végétation submergée pour utiliser celle de la rive... La liaison avec la morphologie végétale apparaît donc assez lâche.

\section{VEGETATION FOURNISSANT UN COU- VERT PROTECTEUR}

\subsection{Espèces recherchant le couvert des buissons}

La biomasse d'une zone couverte de buissons est plus importante que celle d'une futaie sans sousbois de dimensions analogues. Un piégeage dans une Hêtraie-Sapinière dense, dépourvue presque totalement des strates arbustive et herbacée nous a fourni un rendement brut de $4,2 \%$ tandis que, au même moment, le rendement atteignait 21,4\% dans un taillis humide très touffu (Pyrénées-Atlantiques, à une altitude voisine de $1000 \mathrm{~m})$. Certains petits Rongeurs sont si inféodés au couvert des buissons qu'il est exceptionnel de les rencontrer en plein champ. Le bocage de l'ouest de la France constitue un biotope de choix pour l'étude du problème de la liaison des petites espèces avec le couvert végétal. Les talus plantés abritent des populations de Mulots (Apodemus sylvaticus) et de Campagnols roussâtres (Cletbrionomys glareolus) qui ne quittent qu'exceptionnellement l'abri de cette végétation touffue (buissons de ronces ou d'ajoncs, talus plantés d'Ormeaux, de Chênes ou de Noisetiers) et seulement lorsque la végétation est haute dans les parcelles adjacentes. Si la terre est nue après un labour ou si la parcelle est occupée par une prairie pâturée en permanence et donc couverte d'une herbe rase type ",reposoir à bétail", ces deux espèces ne sont jamais capturées à moins de quelques mètres du talus (en général, moins de deux fois la hauteur de la haie). Si la végétation est haute (céréales avant la moisson), elles s'y aventurent quelque peu et le Mulot y établit même des nids temporaires qu'il délaissera immédiatement après la coupe pour revenir au talus. Remarquons également que, lorsque les céréales sont coupées, les Rats des moissons gagnent l'abri des talus plantés ou les hautes herbes des fleurières (bordures de parcelles laissées incultes pour permettre les évolutions des tracteurs ou des animaux de trait).

Une des particularités des populations de talus plantés est aussi la faible dimension de l'espace vital, nettement moins étendu que lorque les mêmes espèces occupent les forêts (Saint Girons, 1976). Il est d'ailleurs intéressant de noter que ces deux phénomènes (nécessité de l'abri du talus et réduction des déplacements) ne sont pas propres aux petits Rongeurs. Ils se manifestent également chez leurs prédateurs directs. C'est le cas des Vipères (Vipera aspis et $V$. berus).

Pour être moins spectaculaire, la liaison des petits Insectivores avec la strate buissonnante n'en est pas moins importante. Fons (1976) a étudié les liens unissant la densité de Crocidura russula et, surtout, Crocidura suaveolens avec la présence du maquis méditerranéen (près de $40 \%$ du total des captures de Crocidures pygmées ont eu lieu dans le maquis, en Roussillon). L'analyse de lots de pelotes de réjection de Tyto alba dans les polders charentais où les formations arbustives sont plutôt rares a montré la faible proportion de Crocidura russula et l'absence de Crocidura suaveolens. Les Campagnols des champs, très fréquents, constituent l'essentiel du régime. D'autres analyses effectuées quelques dizaines de kilomètres plus à l'est dans une zone de bocage ouvert témoignent d'une plus grande abondance de Crocidura russula et de la présence de $C$. suaveolens. Toutes ces données ont été confirmées par le piégeage. Il est toujours difficile de délimiter avec exactitude le rôle imparti à la végétation dans la densité relative des consommateurs secondaires. S'agit-il uniquement du besoin de couvert? N'y a-t-il pas également une richesse et une diversité plus grande de la biomasse des proies autour des buissons? En tout cas, les deux phénomènes semblent agir conjointement pour expliquer la densité des petits Carnivores dans les milieux buissonnants. Ils y trouvent abondance de petits Vertébrés en même temps que protection. C'est ainsi que la Genette, Genetta genetta, vit surtout dans les régions de taillis et évite les grands massifs forestiers (Schauenberg, 1966; Delibes, 1974). Les strates arbustive et arborée peuvent également servir de support à la nourriture des 
Chiroptères. On sait par exemple que les Oreillards (Plecotus auritus et $P$. austriacus) capturent leurs proies non au vol mais posées sur les feuilles, les rameaux ou l'écorce.

Certains Rongeurs semi-aquatiques recherchent également le couvert de la végétation. C'est le cas du Campagnol amphibie, Arvicola sapidus. Nous n'avons repéré ses terriers que le long des berges couvertes de buissons ou d'herbes retombantes.

\subsection{Espèces fuyant le couvert}

Si beaucoup d'espèces recherchent systématiquement le couvert des buissons, d'autres au contraire le fuient et colonisent la strate herbacée découverte. Il s'agit des Mammifères d'origine steppique, consommateurs primaires qui, en Europe de l'ouest, sont essentiellement des Rongeurs de petite taille.

\subsubsection{Nids toujours dans des zones découvertes}

Les petits Campagnols des champs ont toujours leurs nids dans des zones découvertes. Microtus arvalis est caractérisé par une densité très variable par unité de surface, de faibles dimensions de l'espace vital et un taux de reproduction élevé. On ne le rencontre ni dans les talus plantés du bocage, ni dans les formations arbustives non plus que dans les massifs forestiers dépourvus de clairières de quelque importance (Saint Girons, 1974). On sait que le Campagnol des champs est inconnu dans la forêt des Landes alors qu'il pullule sur les deux rives de la Gironde (Saint-Thomas-de-Conac sur la rive droite, Le Verdon-sur-Mer et Cussac sur la rive gauche par exemple). Il constitue la presque totalité du régime de Tyto alba dans les marais d'Aunis ou de Brouage (Charente-Maritime) mais se trouve absent de la forêt de Pins maritimes de la Coubre à l'ouest de Royan où une analyse de pelotes de réjection d'Effraies n'a fourni que 2 exemplaires sur 214 proies, dans un gîte situé à $1500 \mathrm{~m}$ seulement de la lisière de la forêt. Microtus arvalis réapparait en petit nombre dans les clairières cultivées (17 exemplaires sur 97 proies d'Effraies dans une clairière de la forêt de SaintMagrin, une cinquantaine de kilomètres à l'est de la précédente localité) (fig. 2). Sa densité n'atteint toutefois jamais celle des plaines nues voisines. La liaison entre la répartition locale de cette espèce et la présence d'une strate uniquement herbacée
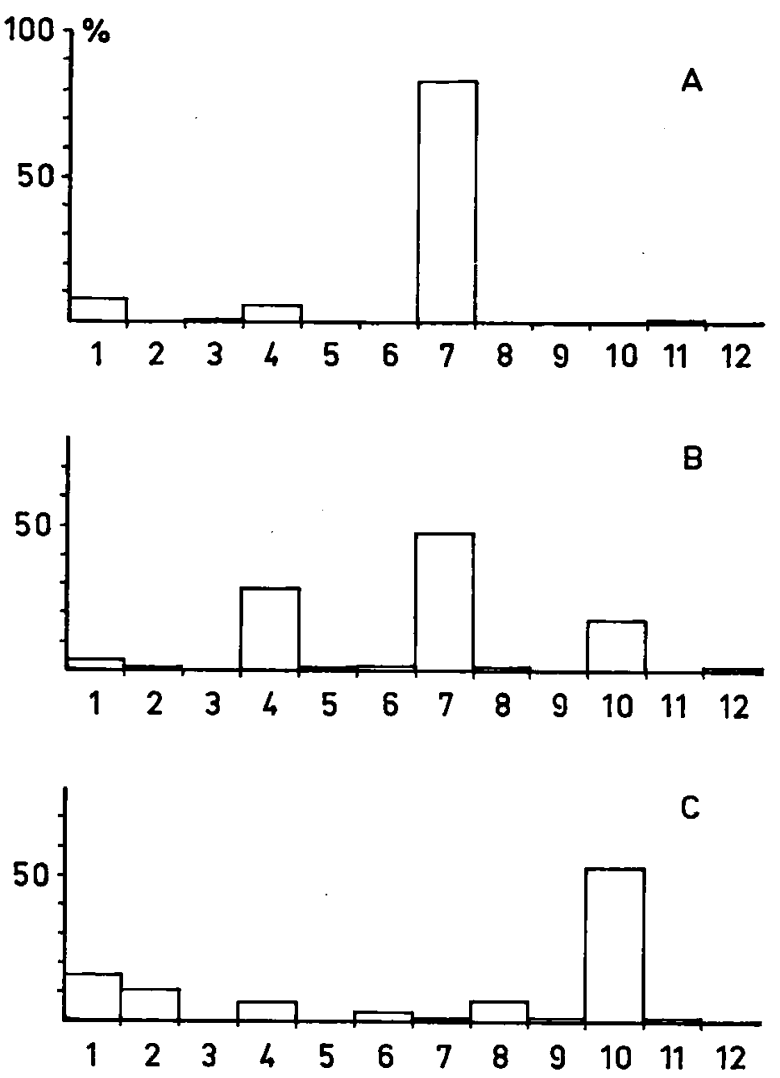

Fig. 2. Résultats d'analyses de pelotes de réjection de Tyto alba en Charente-Maritime. A, Marais d'Aunis; B, Bocage; C, Forêt de la Coubre. En ordonnées le pourcentage des différentes proies, en abscisses les espèces. 1 = Sorex araneus; 2 = Sorex minutus; 3 = Neomys fodiens; $4=$ Crocidura russula; 5 = Crocidura suaveolens; 6 = Clethrionomys glareolus; $7=$ Microtus arvalis; $8=$ Microtus agrestis; $9=$ Arvicola sapidus; $10=$ Apodemus sylvaticus; $11=$ Micromys minutus; 12 = Rattus norvegicus.

est encore plus nette en altitude. Le Campagnol des champs évite les ceintures forestières des étages montagnard et subalpin mais habite les cultures et prairies de fauche des premières pentes aussi bien que les alpages d'altitude. En vallée d'Ossau (Pyrénées-Atlantiques) cette espèce est abondante jusqu'à 500-600 m dans les cultures des fonds de vallée. Aucun individu n'a pu être capturé en Hêtraie-Sapinière, entre 600 et $1500 \mathrm{~m}$, même dans les clairières. L'espèce réapparait vers $1500 \mathrm{~m}$ dans les pâturages au-dessus de la limite des landes à Rhododendrons et dans les fonds de vallées glaciaires en auge (Soussouéou) (fig. 3). C'est donc le Rongeur des prairies naturelles et des friches qui lui assurent un sol suffisamment compact pour ses galeries en même temps qu'une nourriture 


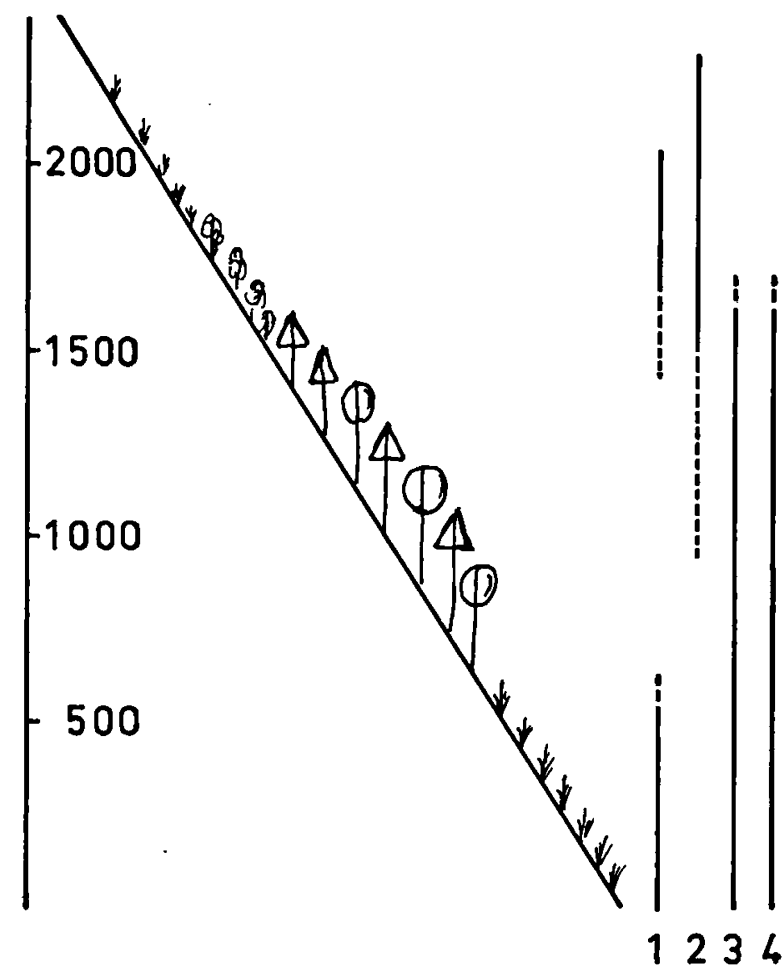

Fig. 3. Répartition en altitude de quelques petits Mammifères dans la vallée d'Ossau (Pyrénées-Atlantiques). Prairies et cultures jusqu'à $500 \mathrm{~m}$, Hêtraie-Sapinière de 500 à $1500 \mathrm{~m}$, landes à Rhododendrons entre 1500 et $1700 \mathrm{~m}$, alpages audessus. $1=$ Microtus arvalis; $2=$ Microtus nivalis; $3=$ Apodemus sylvaticus; $4=$ Cletbrionomys glareolus.

abondante. Pourtant, le rôle trophique de la végétation nous semble réduit. Si l'on en faisait le trait essentiel de la répartition locale de Microtus arvalis, on ne pourrait comprendre son absence des talus du bocage où il trouve un sol profond et bien drainé, jamais bouleversé, en même temps qu'une nourriture qui lui convient dès le pied du talus (fleurière) et même bien souvent sur ses flancs. Sans minimiser l'influence de la compétition avec les Rongeurs mieux adaptés que lui à la vie dans les talus, il nous semble que la présence de la strate arbustive ou arborée constitue un facteur limite négatif de sa répartition locale. Une espèce très voisine, Microtus agrestis, est plus accommodante. Ce Campagnol fréquente de préférence les régions découvertes. Nous l'avons particulièrement étudié en Laponie où, lorsqu'il dispose de biotopes variés et imbriqués, il creuse ses terriers uniquement dans les talus à la limite des cultures ou sous les buissons bordant les prairies et délaisse les bois. En revan- che, si les prairies sont rares, les forêts abritent d'importantes populations de cette espèce (Saint Girons \& Saint Girons, 1970).

L'occupation exclusive ou préférentielle des biotopes découverts se manifeste également chez d'autres Rongeurs des genres Lemmus, Arvicola, Cricetus et Pitymys ainsi que chez le Campagnol nordique, Microtus oeconomus. On peut pourtant trouver les Campagnols souterrains du genre Pitymys dans les vergers ainsi qu'en lisière de bois.

\subsubsection{Nids sous un abri naturel}

Certains petits Rongeurs se nourrissent bien dans la strate herbacée découverte mais creusent leurs galeries dans des endroits plus abrités, en liaison plus ou moins étroite avec les rochers ou les éboulis. C'est le comportement typique d'une espèce très rupicole, Microtus nivalis, dont le biotope est constitué en altitude par la prairie alpine parsemée de nombreux blocs rocheux ainsi que par les pierriers et éboulis colonisés par une végétation ouverte. De même, un Ecureuil terrestre des montagnes marocaines, Atlantoxerus getulus, circule de jour entre les touffes de végétation, ne monte jamais aux arbres et regagne la nuit un abri aménagé parmi les rochers nus. Il est possible que ce comportement soit en liaison avec des besoins tygmotactiques accentués mais en tout cas la présence des strates arbustive et arborée est ici encore un facteur limite négatif de la répartition locale.

\subsection{Espèces forestières}

Il existe enfin des espèces uniquement forestières que l'on ne surprend qu'exceptionnellement hors des grandes forêts considérées comme abri. C'est le cas en particulier de Martes martes, la Martre, et de Felis silvestris, le Chat sauvage. L'un comme l'autre n'ont jamais été capturés dans les zones dénudées ou même les bocages dépourvus de bois de quelque importance. La Martre préfère les forêts de Conifères, peut-être à cause de l'abri permanent fourni par les aiguilles mais aussi de la plus grande fréquence de sa proie essentielle, l'Ecureuil; son actuelle extension dans toute la France est à juste titre mise en liaison avec le remplacement des boisements de feuillus par des Conifères. Quant au Chat sauvage, son extension vers l'ouest à partir des massifs forestiers de l'Europe centrale où il 
demeure assez fréquent semble bel et bien stoppée par l'absence de grands ponts forestiers qui permettraient de passer d'un massif à l'autre (Parent, 1975). Bien entendu, la pression de chasse joue un rôle également important dans ce cas. Les Rongeurs uniquement forestiers sont peu nombreux. On peut citer toutefois le Lemming des forêts, Myopus schisticolor, que nous n'avons jamais rencontré en terrain découvert. Son biotope est constitué par la forêt claire et sèche au sol couvert de mousses et de graminées. Il se nourrit d'ailleurs aux dépens de la strate herbacée (Kalela, 1963) qui joue donc un rôle de renforcement dans le choix de l'habitat.

Dans le cas des espèces forestières, l'absence de l'arbre est donc un facteur limite négatif dans la répartition tant locale que géographique.

\section{VEGETATION SERVANT DE REPERE}

Utilisée comme support pour le nid, matériau de construction ou abri, la végétation joue également un rôle fort peu connu et généralement même insoupçonné qui est celui de repère dans l'espace individuel des animaux, même de très petite taille. Ce paragraphe élargit quelque peu la notion de facteur limite mais il est indispensable de consacrer quelques lignes à ce sujet, ne serait-ce que pour souligner le petit nombre des observations le concernant.

\subsection{Repérage lors du retour au gîte}

Nous avons étudié le problème des repères utilisés dans les expériences de retour au gîte chez deux espèces de petits Rongeurs sylvicoles: Apodemus sylvaticus et Cletbrionomys glareolus (Durup et al., 1973; Saint Girons \& Durup, 1974). Contrairement à ce qui était prévu, les repères apparemment visuels, constitués par les buissons et les arbres ou tout autre obstacle naturel semblent ne jouer aucun rôle dans le retour au gîte en milieu hétérogène. Ceci confirme les résultats de Bovet (1965) sur le Mulot et la Souris en Camargue. Il est d'ailleurs possible que se manifeste un rôle de repère uniquement aux abords immédiats de l'orifice du gite que les individus connaissent parfaitement. Il y a là tout un champ d'observations encore peu prospecté.
Les petits Insectivores dont l'acuité visuelle est encore plus faible que celle des Rongeurs n'ont pas fait l'objet de recherches de ce point de vue, du moins à notre connaissance, à l'exception d'une observation fortuite relative à un Hérisson désertique, Paraechinus aetbiopicus (vide Tranier, 1974). Un individu a tenté de regagner son gîte à l'aide apparemment d'un repérage astral, sans utiliser les repères fournis par les buissons qui parsemaient le biotope.

\subsection{Repérage des chemins régulièrement parcourus} Certains animaux suivent à heure fixe des passées régulières. Leur système „espace-temps” apparaît parfaitement structuré mais on ne connaît pas le plus souvent le mécanisme de cette très grande rigidité (premier trajet fait au hasard puis renforcement à chaque passage?). On sait que certaines espèces de Chauves-Souris, et en particulier Myotis dasycneme, gagnent leurs terrains de chasse en suivant les lignes d'arbres bordant les canaux. La rangée d'arbres constitue un repère. Reconnaître le mécanisme de son action est plus délicat. Ce peut être un repère au sonar, l'écho butant sur un obstacle; peut-être aussi le choix délibéré d'itinéraires où les Insectes sont nombreux (Myotis dasycneme chasse à la surface de l'eau) ou encore l'utilisation des turbulences créées par l'augmentation de la rugosité au vent ou la chûte de la température à l'aplomb des lignes d'arbres. Les bordures forestières, les allées dégagées constituent aussi des itinéraires régulièrement utilisés par plusieurs espèces de Chauves-Souris.

\section{EXCEPTIONS ET ADAPTATIONS}

Tout ce qui précède tendrait à montrer que les petits Mammifères manifestent des liens d'une grande rigidité vis-à-vis de la végétation, ce qui sous-entendrait une faible adaptabilité comportementale. Ce n'est pas tout à fait exact. En fait, cette influence de la végétation s'exerçant de façon rigoureuse est loin de se manifester chez toutes les espèces. Des Rongeurs occupent des niches écologiques variées: Eliomys quercinus gîte dans les arbres creux mais aussi dans les anfractuosités des grottes ou les constructions. Il en est de même du Loir, Glis glis. Dans ces deux cas, on peut estimer que ce qui importe pour l'animal c'est de placer son 
gîte dans un endroit sombre, aux parois dures et imperméables, assurant des conditions microclimatiques quasi constantes. Peu importe le matériau dans lequel il est creusé. De même, beaucoup de Rongeurs non hibernants utilisent les constructions comme abri (Apodemus, Clethrionomys, Microtus arvalis). Les Chauves-Souris cavernicoles gitent indifféremment l'hiver dans les galeries souterraines ou les caves de bâtiments. Microtus nivalis, rupicole typique, vit apparemment très bien dans les chalets d'altitude. Les petits Mammifères souterrains (Talpa) et aquatiques (Neomys) sont à peu près indépendants de la morphologie végétale ... Il est donc difficile de considérer la morphologie du couvert végétal comme un facteur limite très impératif de la répartition locale. Ceci ne s'applique qu'à quelques cas bien précis où la végétation sert obligatoirement de soutien ou de matériau pour le nid ainsi que d'abri contre les prédateurs y compris l'Homme. On n'imagine pas l'Ecureuil, la Martre ou le Chat sauvage dans un milieu dépourvu d'arbres, le Muscardin sur une prairie rase ou encore le Rat des moissons dans une futaie sans sous-bois. Pourtant, en dehors de ces exemples, somme toute assez peu nombreux, le facteur limite doit être cherché ailleurs (conditions nutritionnelles ou édaphiques, concurrence, prédation, etc...) et peut bien entendu varier d'une région à l'autre. Microtus arvalis est absent des zones forestières du sud-ouest mais ce sont des facteurs climatiques qui limitent sa répartition dans la basse vallée du Rhône, la faible profondeur $\mathrm{du}$ sol dans les terrains calcaires du Vercors et du Périgord, les pratiques culturales jointes à l'humidité du climat dans l'ouest complètement déboisé, ou encore la faible profondeur de la nappe phréatique dans les Hautes Fagnes de l'Ardenne. On pourrait multiplier les exemples de ce genre. Même les Castors sont capables de renoncer aux huttes et aux barrages pour utiliser le réseau hydrographique souterrain ou creuser des terriers.

Le peuplement des îles apporte une autre preuve de l'adaptabilité des Mammifères. Certaines d'entre elles n'ont pas été peuplées par toutes les espèces continentales de telle sorte que telle niche écologique „devrait" théoriquement rester libre. Or, il n'en est rien. Le cas des îles de la Manche et de l'Atlantique a été maintes fois cité. Trois petits
Rongeurs, Microtus arvalis, Microtus agrestis et Cletbrionomys glareolus sont répartis de telle sorte qu'un seul d'entre eux, tantôt l'un, tantôt l'autre, se trouve représenté dans chaque île. Or le Campagnol des champs, Rongeur des champs ouverts, et le Campagnol roussâtre, espèce des forêts et des talus plantés, sont inféodés sur le continent à deux niches écologiques très différentes et ne s'y rencontrent jamais ensemble. De même, la Crossope, Neomys fodiens, seul Insectivore présent dans l'île de Texel (Pays-Bas) y occupe tous les biotopes y compris les dunes de sable fréquentées uniquement par les Musaraignes (Sorex araneus) sur le continent. Il semble bien que, en l'absence de concurrence, la première espèce qui prenne pied dans une île occupe tous les biotopes sans se soucier du couvert végétal. Ensuite, la concurrence interspécifique joue, éliminant les représentants des autres espèces arrivées plus tard, quand toutes les niches écologiques étaient déjà occupées (Cameron, 1964; Saint Girons \& Beaucournu, 1970).

A l'aide d'exemples, nous avons voulu attirer l'attention sur le rôle joué par la morphologie végétale dans la vie des Mammifères. Ce rôle a trop souvent été minimisé au profit de celui de la végétation considérée comme source de nourriture. $\mathrm{La}$ morphologie végétale n'est toutefois un facteur limite absolu que dans un très petit nombre de cas. Nous pensons qu'elle joue surtout un rôle dans la répartition locale des populations dans les niches écologiques disponibles et ceci le plus souvent par le biais des pratiques culturales. La densité locale des espèces en dépend aussi grandement. La liaison est plus étroite entre la morphologie végétale et les consommateurs primaires (Rongeurs) et s'avère moins apparente pour les Mammifères prédateurs, sauf lorsqu'il s'agit d'espèces d'assez grande taille qui s'abritent sous un couvert épais. Au niveau de l'aire de répartition des espèces, ce sont des facteurs géographiques qui jouent le plus souvent le rôle de facteurs limites.

Nous remercions ici tous nos correspondants qui nous font parvenir des pelotes de réjection de Rapaces et particulièrement Monsieur A. Doumeret qui a entrepris l'étude systématique de la Charente-Maritime. Notre travail en montagne doit beaucoup à l'aide de nos collègues A. Fayard, R. Fons, R. Libois et F. Turpin. Enfin, notre collègue et ami P. J. H. van Bree a accepté de relire ce manuscrit et d'en traduire le résumé. Nous tenons à l'en remercier ici. 


\section{BIBLIOGRAPHIE}

Bovet, J., 1965. Note sur le retour au gite du Mulot de Camargue. Z. Tierpsych., 22: 163-166.

Cameron, A. W., 1964. Competitive exclusion between the rodent genera Microtus and Clethrionomys. Evolution, 18: $630-634$.

Darchen, R. \& J. Lecomte, 1959. Sur la signification des huttes d'hiver d'Ondatra zibethica L. C. R. Acad. Sci., 248: 1046-1048.

Durup, M., M. C. Saint Girons, C. Fabrigoule \& H. Durup, 1973. Quelques données sur les modalités du retour au gîte chez le Mulot, Apodemus sylvaticus, et le Campagnol roussâtre, Clethrionomys glareolus. Mammalia, 37: 33-55.

Delibes, M., 1974. Sobre alimentacion y biologia de la Gineta (Genetta genetta L.) en España. Donana, (A) (Vertebrata) 1: 143-199.

Fons, R., 1976. Premières données sur l'écologie de Suncus etruscus (Savi, 1822). Comparaison avec deux autres Crocidurinae Crocidura russula (Hermann, 1780) et Crocidura suaveolens (Pallas, 1811). Insectivora, Soricidae. Vie Milieu, (C) 25 (2): 315-360.

JENNINGS, T. J., 1975. Notes on the burrow systems of woodmice (Apodemus sylvaticus). J. Zool., London, 177: $500-504$.

KaнmanN, H., 1951. Die deutschen Schlafmäuse. Kosmos, 47: 491.496.

KAlelA, O., 1963. Beiträge zur Biologie des Waldlemmings Myopus schisticolor (Lillj.). Arch. Soc. Vanamo, Helsinki, Suppl. 18: 1-96.

Koenig, L., 1960. Das Aktionssystem des Siebenschläfers (Glis glis L.). Z. Tierpsych., 17: 427-505.

Meeuwissen, E., 1948. Observation sur le Rat musqué, Ondatra zibethica (Linné) dans le Limbourg belge. Bull. Mus. roy. Hist. nat. Belg., 24 (32): 1-8.

Parent, G. H., 1975. La migration récente à caractère inva- sionnel du Chat sauvage Felis silvestris silvestris Schreber, en Lorraine belge. Mammalia, 39: 251-288.

Petter, F., 1953. Note préliminaire sur l'éthologie et l'écologie de Meriones libycus (Rongeurs, Gerbillidés). Mammalia, 17: 281-294.

RICHARD, P. B., 1962. Le comportement constructeur du Rat musqué (Ondatra zibethica) comparé à celui du Castor (Castor fiber). J. Psychol., 1962: 405-412.

_-1964. Les matériaux de construction du Castor (Castor fiber). Leur signification pour ce Rongeur. Z. Tierpsych., 21: $592-601$.

SAINT Girons, M. C., 1974. Les petits Mammifères de France et leurs biotopes. In „Recherches biologiques contemporaines": 391-401 (L. Arvy; Nancy, Vagner éd.).

, 1976. Les petits Mammifères dans l'écosystème bocager. C. R. Colloque C.N.R.S. „Ecosystèmes bocager”. (Rennes, Juillet 1976). (Sous presse).

Saint Girons, M. C. \& J. C. Benucournu, 1970. Notes sur les Mammifères de France, 10 - Le Campagnol roussâtre de Belle-Isle (Morbihan), Clethrionomys glareolus insulaebellae Heim de Balsac, 1940. Comparaison avec une population continentale proche, Clethrionomys glareolus glareolus (Schreber, 1780) de Puceul (Loire-Atlantique). Mammalia, 34: 617-621.

SAINT Girons, M. C. \& M. Durup, 1974. Retour au gite chez le Mulot, Apodemus sylvaticus, et le Campagnol roussâtre, Clethrionomys glareolus. Facteurs écologiques, apprentissage et mémoire. Mammalia, 38: 389.404 .

SaINT Girons, M. C. \& H. Saint Girons, 1970. Données sur l'écologie des petits Mammifères en Laponie. Terre Vie, 117: 331-345.

Schaunnberg, P., 1966. La Genette vulgaire (Genetta genetta L.); répartition géographique en Europe. Mammalia, 30: 371.396.

Tranier, M., 1974. Retour au gîte d'un Hérisson du désert, Paraechinus aethiopicus. Mammalia, 38: 749-751.

Reçu le 3 décembre 1976 